\title{
TNFRSF8 Low
}

National Cancer Institute

\section{Source}

National Cancer Institute. TNFRSF8 Low. NCI Thesaurus. Code C131485.

Indicates that low expression of TNFRSF8 has been detected in a sample. 